Linköping Studies in Science and Technology.

Dissertations No. 1825

\title{
Families of Sets Without the Baire Property
}

\section{Venuste NYAGAHAKWA}

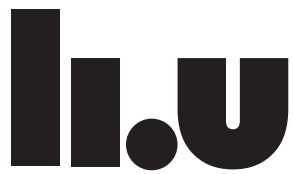

LINKÖPING UNIVERSITY

Department of Mathematics

Division of Mathematics and Applied Mathematics

Linköping University, SE-581 83 Linköping, Sweden

Linköping 2017 
Linköping Studies in Science and Technology.

Dissertations No. 1825

\section{Families of Sets Without the Baire Property}

Venuste NYAGAHAKWA

venuste.nyagahakwa@liu.se

www.mai.liu.se

Mathematics and Applied Mathematics

Department of Mathematics

Linköping University

SE-581 83 Linköping

Sweden

ISBN 978-91-7685-592-8ＩSSN 0345-7524

Copyright (C) 2017 Venuste NYAGAHAKWA

Printed by LiU-Tryck, Linköping, Sweden 2017 
To Rose, Roberto and Norberto 



\section{Abstract}

The family of sets with the Baire property of a topological space $X$, i.e., sets which differ from open sets by meager sets, has different nice properties, like being closed under countable unions and differences. On the other hand, the family of sets without the Baire property of $X$ is, in general, not closed under finite unions and intersections. This thesis focuses on the algebraic set-theoretic aspect of the families of sets without the Baire property which are not empty. It is composed of an introduction and five papers.

In the first paper, we prove that the family of all subsets of $\mathbb{R}$ of the form $(C \backslash M) \cup N$, where $C$ is a finite union of Vitali sets and $M, N$ are meager, is closed under finite unions. It consists of sets without the Baire property and it is invariant under translations of $\mathbb{R}$. The results are extended to the space $\mathbb{R}^{n}$ for $n \geq 2$ and to products of $\mathbb{R}^{n}$ with finite powers of the Sorgenfrey line.

In the second paper, we suggest a way to build a countable decomposition $\left\{X_{i}\right\}_{i=1}^{\infty}$ of a topological space $X$ which has an open subset homeomorphic to $\left(\mathbb{R}^{n}, \tau\right), n \geq 1$, where $\tau$ is some admissible extension of the Euclidean topology, such that the union of each non-empty proper subfamily of $\left\{X_{i}\right\}_{i=1}^{\infty}$ does not have the Baire property in $X$. In the case when $X$ is a separable metrizable manifold of finite dimension, each element of $\left\{X_{i}\right\}_{i=1}^{\infty}$ can be chosen dense and zero-dimensional.

In the third paper, we develop a theory of semigroups of sets with respect to the union of sets. The theory is applied to Vitali selectors of $\mathbb{R}$ to construct diverse abelian semigroups of sets without the Baire property. It is shown that in the family of such semigroups there is no element which contains all others. This leads to a supersemigroup of sets without the Baire property which contains all these semigroups and which is invariant under translations of $\mathbb{R}$. All the considered semigroups are enlarged by the use of meager sets, and the construction is extended to Euclidean spaces $\mathbb{R}^{n}$ for $n \geq 2$.

In the fourth paper, we consider the family $\mathcal{V}_{1}(Q)$ of all finite unions of Vitali selectors of a topological group $G$ having a countable dense subgroup $Q$. It is shown that the collection $\left\{G \backslash U: U \in \mathcal{V}_{1}(Q)\right\}$ is a base for a topology $\tau(Q)$ on $G$. The space $(G, \tau(Q))$ is $T_{1}$, not Hausdorff and hyperconnected. It is proved that if $Q_{1}$ and $Q_{2}$ are countable dense subgroups of $G$ such that $Q_{1} \subseteq Q_{2}$ and the factor group $Q_{2} / Q_{1}$ is infinite (resp. finite) then $\tau\left(Q_{1}\right) \nsubseteq \tau\left(Q_{2}\right)$ (resp. $\tau\left(Q_{1}\right) \subseteq$ $\left.\tau\left(Q_{2}\right)\right)$. Nevertheless, we prove that all spaces constructed in this manner are homeomorphic.

In the fifth paper, we investigate the relationship (inclusion or equality) between the families of sets with the Baire property for different topologies on the same underlying set. We also present some applications of the local function defined by the Euclidean topology on $\mathbb{R}$ and the ideal of meager sets there. 



\section{Populärvetenskaplig sammanfattning}

Den familj av mängder som har Baire-egenskapen i ett topologiskt rum $X$, det vill säga mängder som är differensen mellan en öppen och en mager mängd, är som bekant en $\sigma$-algebra. Däremot är familjen av mängder som inte har Baireegenskapen i $X$ i allmänhet inte sluten under någon av de vanliga mängdoperationerna. Denna avhandling, bestående av en inledning och fem artiklar, handlar om de algebraiskt mängdteoretiska aspekterna hos familjen av mängder som inte har Baire-egenskapen.

I den första artikeln visar vi att familjen av delmängder av rummet $\mathbb{R}$ av formen $(C \backslash M) \cup N$, där $C$ är en ändlig union av Vitali-mängder och $M$ och $N$ är magra, är sluten under ändliga unioner. Familjen består av mängder utan Baire-egenskapen och den är invariant under translationer. Vi utvidgar också dessa resultat till rum som är ändliga produkter av $\mathbb{R}$ och Sorgenfrey-linjen.

I den andra artikeln konstruerar vi, för ett topologiskt rum $X$ som har en öppen delmängd homeomorf med $\left(\mathbb{R}^{n}, \tau\right)$, där $\tau$ är en tillåten utvidgning av den euklidiska topologin, en uppräknelig uppdelning $\left\{X_{i}\right\}_{i=1}^{\infty}$ av $X$ sådan att unionen av varje icketom äkta delfamilj inte har Baire-egenskapen i $X$. Om $X$ är en ändligdimensionell, separabel och metriserbar mångfald så kan varje $X_{i}$ väljas att vara tät och nolldimensionell.

I den tredje artikeln utvecklar vi en teori för semigrupper av mängder, med union som gruppoperation, och tillämpar denna teori på Vitali-selektorer i $\mathbb{R}$ för att konstruera olika semigrupper av mängder som inte har Baire-egenskapen. Vi konstruerar sedan en semigrupp av mängder som innehåller alla dessa semigrupper och som består av mängder utan Baire-egenskapen och är invariant under translationer. Semigrupperna kan utvidgas med hjälp av magra mängder, och vi generaliserar resultaten till rummet $\mathbb{R}^{n}$.

I den fjärde artikeln betraktar vi familjen $\mathcal{V}_{1}(Q)$ bestående av ändliga unioner av Vitali-selektorer i en topologisk grupp $G$ med en uppräknelig tät delgrupp $Q$. Vi visar att $\left\{G \backslash U: U \in \mathcal{V}_{1}(Q)\right\}$ är en bas för en topologi $\tau(Q)$ på $G$. Rummet $(G, \tau(Q))$ är $T_{1}$ och hypersammanhängande men inte Hausdorff. Vi visar också att om $Q_{1}$ och $Q_{2}$ är uppräkneliga täta delgrupper av $G$ sådana att $Q_{1} \subseteq Q_{2}$ och kvotgruppen $Q_{2} / Q_{1}$ är oändlig (respektive ändlig) så gäller att $\tau\left(Q_{1}\right) \nsubseteq \tau\left(Q_{2}\right)$ (respektive $\tau\left(Q_{1}\right) \subseteq \tau\left(Q_{2}\right)$ ). Vi visar dock att alla topologiska rum konstruerade på detta sätt är homeomorfa.

I den femte artikeln undersöker vi relationen mellan familjerna av mängder med Baire-egenskapen för olika topologier på samma underliggande mängd. Vi ger också några tillämpningar av den lokala funktionen hörande till den euklidiska topologin på $\mathbb{R}$ och idealet av magra mängder. 



\section{Acknowledgments}

This thesis appears in its current form due to the assistance, and guidance from several people. Therefore, I would like to express my sincere thanks to them.

Foremost, I wish to express my gratitude to my main supervisor Vitalij A. Chatyrko for useful comments, remarks and encouragement throughout my graduate studies. Thank you Vitalij for helping me to improve my writing skills, and for your willingness to provide me advice about my future career.

I also express my gratitude to my assistant supervisor Mats Aigner for his helpful collaboration. All insightful discussions we had together gave me additional energy for conducting research. I am also grateful to my second assistant supervisor Isidore Mahara whom I worked with every time I went back to Rwanda.

In a very special way, I thank Bengt Ove Turesson, Björn Textorius, Martin Singull, Theresa Lagali Hensen, Meaza Abebe and Aksana Mushkavet for their invaluable assistance and support. I thank Tomas Sjödin, Theresia Carlsson Roth, Hans Lundmark and all members of the Department of Mathematics for building up and maintaining a high-quality working environment and for their help and support whenever needed at work.

I am truly grateful to Froduald Minani and Alexandre Lyambabaje who not only taught me Mathematics but also encouraged and supported me in different kinds of academic activities. My gratitude also goes to the staff members of the UR-Sweden Programme for Research, Higher Education and Institutional Advancement, for their contribution to the smooth running of my studies.

I am thankful to my fellow Ph.D students whom I shared so many excellent times at Linköping University. Thanks are also due to Jolanta Maria Pielaszkiewicz for having organized a wonderful trip to visit some historical sites in Poland.

I am eternally grateful to my family and friends who supported and helped me along my studies by giving encouragement and providing the moral and emotional support when needed.

I gratefully acknowledge the financial support received from the Swedish International Development Cooperation Agency funded through Linköping University and the University of Rwanda.

Finally, I extend my sincere thanks to other people and institutions who supported me in one way or another.

May the Lord God bless all of you. 



\section{List of papers}

This thesis is based on the following appended papers and they are referred by their roman numerals.

I. V. A. Chatyrko and V. Nyagahakwa, On the families of sets without the Baire property generated by the Vitali sets, p-Adic Numbers, Ultrametric Analysis, and Applications, 2011, Vol.3, No.2, 100 - 107.

II. M. Aigner, V. A. Chatyrko and V. Nyagahakwa, On countable families of sets without the Baire property, Colloquium Mathematicum, 2013, Vol.133, No.2, 179 - 187.

III. M. Aigner, V. A. Chatyrko, and V. Nyagahakwa, The algebra of semigroups of sets, Mathematica Scandinavica, 2015, Vol.116, No.2, 161 - 170.

IV. V. A. Chatyrko and V. Nyagahakwa, Vitali selectors in topological groups and related semigroups of sets, Questions and Answers in General Topology, 2015, Vol.33, No.2, 93 - 102.

V. V. A. Chatyrko and V. Nyagahakwa, Sets with the Baire property in topologies formed from a given topology and ideals of sets, Accepted for publication in Questions and Answers in General Topology.

The research questions and results presented in the above papers were the product of multiple discussions between the authors. In Papers I and II, I was actively involved in discussions about the research subjects and the structure of the papers. For Paper III, all the three authors jointly contributed to writing 
and deriving the results. In particular, I developed the part about the algebra of semigroups and ideals of sets. The results in Paper IV were developed jointly with my co-author. I wrote the part about diversity of semigroups of Vitali selectors. Regarding Paper V, I formulated the research topic and wrote the first draft. The final form of the paper was reached through discussions with my co-author.

Some parts of this thesis have been presented by me at the following conferences or seminars.

1. International Conference on Topology: On the Occasion of Filippo Cammaroto's 65th Birthday, Messina-Italy, 7 - 11 September 2015.

2. The third International Modelling Week, University of Rwanda, College of Science and Technology, Kigali-Rwanda, 25 - 29 August 2014.

3. Analysis seminar \& Mathematical Colloquium, Linköping University-Sweden, 2013, 2014, 2015, 2016.

4. The third EAUMP Conference: Advances in Mathematics and its Applications, Makerere University, Kampala-Uganda, 26 - 27 October 2016. 


\section{Contents}

\begin{tabular}{lll}
\hline I & BACKGROUND AND SUMMARY & 1
\end{tabular}

$\begin{array}{lll}1 & \text { Introduction } & 3\end{array}$

1.1 Statement of the problem . . . . . . . . . . . . . . 4

1.2 Aim of the thesis $\ldots \ldots \ldots \ldots \ldots$

\begin{tabular}{lll}
\hline 2 & Background & 9
\end{tabular}

$2.1 \quad$ Algebraic notions in Set Theory $\ldots \ldots \ldots \ldots$

2.2 Sets with the Baire property $\ldots \ldots \ldots \ldots \ldots \ldots$

2.3 Elements of topological groups $\ldots \ldots \ldots \ldots \ldots$

2.4 Topological products of an infinite family of spaces . . . . . . . . 14

2.5 Extension of topologies via ideals . . . . . . . . . . . . . 14

2.6 Admissible extensions of topologies . . . . . . . . . . . . . 16

2.7 Vitali selectors on the real line . . . . . . . . . . . . . . . 17

2.8 Lebesgue covering dimension $\ldots \ldots \ldots \ldots$



\begin{tabular}{ll}
\hline Bibliography & 27
\end{tabular} 
II INCLUDED PAPERS $\quad 29$

I On the families of sets without the Baire property generated by $\begin{array}{ll}\text { the Vitali sets } & 31\end{array}$

$1 \quad$ Introduction . . . . . . . . . . . . . . . . . . . . . 33

2 Preliminary facts . . . . . . . . . . . . . . . . . . . 34

3 Main results . . . . . . . . . . . . . . . . . . . . 35

4 Concluding remarks . . . . . . . . . . . . . . . . . . . . . . . 42

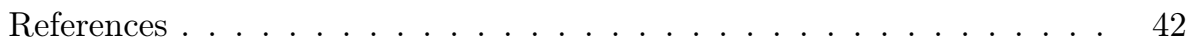

II On countable families of sets without the Baire property 45

$1 \quad$ Introduction . . . . . . . . . . . . . . . . . . . . . 47

2 Auxiliary results . . . . . . . . . . . . . . . . . . . . 48

3 A method of constructing countable families of sets without the

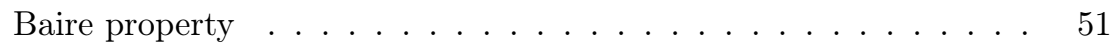

4 Concluding remarks . . . . . . . . . . . . . . . . . 54

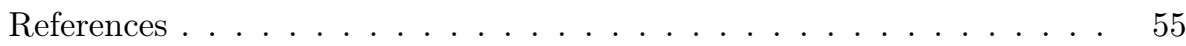

\begin{tabular}{ll}
\hline III The algebra of semigroups of sets & 57
\end{tabular}

1 $\quad$ Introduction . . . . . . . . . . . . . . . . . . . . . . . . . 59

2 Auxiliary notions . . . . . . . . . . . . . . . . . 60

3 Semigroups of sets and ideals of sets . . . . . . . . . . . . . . . 60

4 Applications. . . . . . . . . . . . . . . . . 64

$4.1 \quad$ Two nested families of semigroups of sets . . . . . . . . 64

$4.2 \quad$ Supersemigroups based on the Vitali sets . . . . . . . . . . 65

$4.3 \quad$ A nonmeasurable case . . . . . . . . . . . . . . . . 67

References. . . . . . . . . . . . . . . . . 67

IV Vitali selectors in topological groups and related semigroups of $\begin{array}{ll}\text { sets } & 69\end{array}$

$1 \quad$ Introduction . . . . . . . . . . . . . . . . . . . . 71

2 Auxiliary notions . . . . . . . . . . . . . . . . 73

$2.1 \quad$ Baire property . . . . . . . . . . . . 73

$2.2 \quad$ Semigroups and ideals of sets . . . . . . . . . . . . 73

$2.3 \quad$ A topology defined by a semigroup . . . . . . . . . . . . . 74 
$3 \quad$ Vitali selectors in an abelian topological group $G$ and related semigroup of sets ...................... 74

$3.1 \quad$ A semigroup of Vitali selectors of $G$. . . . . . . . . . . . 75

3.2 Diversity of semigroups of Vitali selectors . . . . . . . . . . 76

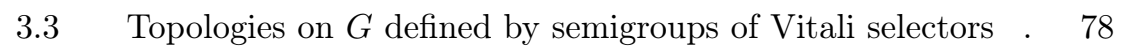

References . . . . . . . . . . . . . . . . . . 80

\begin{tabular}{|l|}
\hline V Sets with the Baire property in topologies formed from a given \\
\hline \hline topology and ideals of sets
\end{tabular}

1 Introduction . . . . . . . . . . . . . . . . . . 85

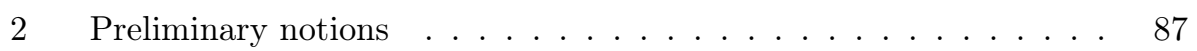

$2.1 \quad$ Ideal of sets and the Baire property . . . . . . . . . . . . . 87

$2.2 \quad$ Local functions and Kuratowski operators via ideals of sets, *-topologies . . . . . . . . . . . . . . . . 88

$2.3 \quad$ Some natural relation between topologies and ideals . . . . 88

$3 \quad$ Two topologies on the same set and the Baire property . . . . . . . 89

$3.1 \quad \pi$-compatible topologies . . . . . . . . . . . . . . . . . . 89

$3.2 \quad$ A special case of $\pi$-compatible topologies . . . . . . . . . . 90

3.3 Codense ideals of sets and sets with the Baire property . . 92

$3.4 \quad$ Partial equality of the families of nowhere dense sets (resp. meager sets or sets with the Baire property) . . . . . . 95

$4 \quad$ Three and more topologies on the same set and the Baire property 96

$4.1 \quad$ Ideals of sets producing equal $*$-topologies . . . . . . . . . . 96

$4.2 \quad$ Ideals of sets producing different $*$-topologies . . . . . . . . 98

5 Applications of the local function for proving some known facts . . 100

$5.1 \quad$ Applications to Bernstein sets . . . . . . . . . . . . . . . . 100

$5.2 \quad$ Applications to Vitali sets . . . . . . . . . . . . . . . . . 101

References . . . . . . . . . . . . . . . . . 102 

Part I

\section{BACKGROUND AND SUMMARY}



Introduction

In the early decades of the twentieth century, the work of French analysts E. Borel, R. Baire and H. Lebesgue provided the foundations of modern measure and integration theory as well as descriptive set theory. In their respective doctoral theses, they introduced the fundamental concepts related to measurability and category on the real line. Ever since, these concepts and their generalizations have been subject to intense investigation.

In his thesis, "Sur quelques points de la théorie des fonctions", (1894), Borel created the first effective theory of measure [FK], extending the notion of length of intervals to a measure on a wide class of subsets of $\mathbb{R}$. Borel considered measurable sets to be those subsets of $\mathbb{R}$ which are obtained by starting with intervals and forming the closure with respect to complementations and countable unions. The family of such sets is a $\sigma$-algebra and its elements are known as the Borel sets or the Borel-measurable sets on the real line.

A few years later, Baire introduced basic concepts related to category and set up a classification of functions [GW]. In his thesis "Sur les fonctions de variables réelles", (1899), Baire defined nowhere dense sets, first category sets and second category sets on the real line, and proved the "Baire Category Theorem", a fundamental theorem in topology and functional analysis. Furthermore, he introduced the concept known today as the "Baire property". A set with the Baire property is a set which differs from an open set by a set of first category. The family of sets with the Baire property is a $\sigma$-algebra on $\mathbb{R}$ which contains all Borel sets as well as all first category sets on the real line.

Inspired by Borel's results, Lebesgue further extended the family of measur- 
able sets on the real line [GW]. In his thesis, "Intégrale, longueur, aire", (1902), Lebesgue defined the outer measure of a subset $E$ of $\mathbb{R}$ as the infimum, taken over all sequences of intervals covering $E$, of their total lengths. He also defined the inner measure of a set by using the outer measure and the operation of complement of sets. He then defined a set to be measurable if its outer measure equalled its inner measure, and the Lebesgue measure as their common value. In this way, he obtained a complete measure extending the Borel measure. The family of all Lebesgue measurable sets on $\mathbb{R}$ is a $\sigma$-algebra which contains all Borel-measurable subsets of the real line.

In 1905, using the Axiom of Choice, the Italian mathematician G. Vitali constructed a first example of a subset of $\mathbb{R}$ which is neither Lebesgue measurable nor has the Baire property [Vi]. This set is known today as the Vitali set and its appearance has stimulated further developments in mathematics. Apart from Vitali sets, there exist other types of subsets of $\mathbb{R}$ (for example Bernstein sets and sets associated with Hamel bases) which are neither Lebesgue measurable nor have the Baire property $\mathrm{Ox}, \mathrm{Kh1}$. In fact the literature on such kind of sets is huge.

We note that there exists a model of set theory in which every subset of the real line is Lebesgue measurable and has the Baire property. Indeed, in 1970, Solovay [So showed that the existence of a non-Lebesgue measurable subset of the real line (or the existence of a subset of the real line without the Baire property) is unprovable if the Axiom of Choice is disallowed.

\subsection{Statement of the problem}

Consider the set $\mathbb{R}$ of real numbers endowed with the Euclidean topology and denote by $\mathscr{P}(\mathbb{R})$ the family of all subsets of $\mathbb{R}$. Let $\mathscr{B}_{p}$ be the subfamily of $\mathscr{P}(\mathbb{R})$ consisting of sets with the Baire property in $\mathbb{R}$ and let $\mathcal{L}$ be the subfamily of $\mathscr{P}(\mathbb{R})$ consisting of Lebesgue measurable subsets of $\mathbb{R}$. The families $\mathscr{B}_{p}$ and $\mathcal{L}$ have a number of similarities. Namely, they are $\sigma$-algebras of sets on $\mathbb{R}$ (and hence they are closed under all basic set operations) and they are invariant under the action of the group $\tau(\mathbb{R})$ of all translations of $\mathbb{R}$, i.e., if $A \in \mathscr{B}_{p}$ (resp. $\left.A \in \mathcal{L}\right)$ and $h \in \tau(\mathbb{R})$ then $h(A) \in \mathscr{B}_{p}$ (resp. $h(A) \in \mathcal{L}$ ). As a $\sigma$-algebra, the family $\mathscr{B}_{p}$ is generated by the open sets of $\mathbb{R}$ together with the $\sigma$-ideal of all first category sets on $\mathbb{R}$ while the family $\mathcal{L}$ is generated by the open sets of $\mathbb{R}$ together with the $\sigma$-ideal of all subsets of $\mathbb{R}$ having the Lebesgue measure zero. Therefore, each of the families $\mathscr{B}_{p}$ and $\mathcal{L}$ contains the $\sigma$-algebra of all Borel sets of the real line.

As mentioned above, the complement $\mathscr{B}_{p}^{c}$ of $\mathscr{B}_{p}$ in $\mathscr{P}(\mathbb{R})$, as well as the complement $\mathcal{L}^{c}$ of $\mathcal{L}$ in $\mathscr{P}(\mathbb{R})$, is non-empty. In fact, each Vitali set, as well as each Bernstein set, does not have the Baire property on the real line and it is not measurable in the Lebesgue sense.

Since each subset of $\mathbb{R}$ can be decomposed into a disjoint union of a first 
category set and a set having the Lebesgue measure zero $[\mathrm{Ox}$, none of the families $\mathscr{B}_{p}$ and $\mathcal{L}$ contains the other. More precisely, if we decompose a Vitali subset of $\mathbb{R}$ (or a Bernstein subset of $\mathbb{R}$ ) into a disjoint union of a meager set and a null set, then one of the sets in this decomposition will be non-Lebesgue measurable but with the Baire property, and the other will be Lebesgue measurable but without the Baire property.

It also follows that none of the families $\mathscr{B}_{p}^{c}$ and $\mathcal{L}^{c}$ contains each other. However, unlike $\mathscr{B}_{p}$ and $\mathcal{L}$, the families $\mathscr{B}_{p}^{c}$ and $\mathcal{L}^{c}$ are not closed under basic set operations.

The intensive study of the families $\mathscr{B}_{p}^{c}$ and $\mathcal{L}^{c}$ was mostly concentrated on the construction of their elements with peculiar properties (cf. [Kh1]). For example, there are subgroups $G$ of the additive group $(\mathbb{R},+)$ of reals which are Bernstein sets (and hence such $G$ belong to both families $\mathscr{B}_{p}^{c}$ and $\mathcal{L}^{c}$ ) and for which cardinality of the factor group $(\mathbb{R},+) / G$ is continuum. However, this thesis was stimulated by the following question, posed by Chatyrko:

Do there exist rich subfamilies of $\mathscr{B}_{p}^{c}$ (respectively, $\mathcal{L}^{c}$ ), which are invariant under the action of an infinite subgroup of the group $\mathscr{H}(\mathbb{R})$ of all homeomorphisms of $\mathbb{R}$ and on which we can define some algebraic structure from the set-theoretic point of view?

One answer to this question was given by Chatyrko in Ch1 where he showed that each finite union of Vitali sets does not possess the Baire property on the real line. He also observed that the family $\mathcal{S}_{\mathcal{V}}$ of all finite unions of Vitali sets is invariant under translations of $\mathbb{R}$. From these facts, it follows that the family $\mathcal{S}_{\mathcal{V}}$ is an abelian semigroup of sets with respect to the operation of union of sets, that it is invariant under translations of $\mathbb{R}$, and that $\mathcal{S}_{\mathcal{V}} \subseteq \mathscr{B}_{p}^{c}$.

Furthermore, it can be shown that the dual family $\mathcal{S}_{\mathcal{V}}^{*}=\left\{\mathbb{R} \backslash S: S \in \mathcal{S}_{\mathcal{V}}\right\}$ is also an abelian semigroup of sets with respect to the operation of intersection of sets, that it is invariant under translations of $\mathbb{R}$, and that $\mathcal{S}_{\mathcal{V}}^{*} \subseteq \mathscr{B}_{p}^{c}$.

Similarly, in Kh2] Kharazishvili showed that each finite union of Vitali sets is not measurable in the Lebesgue sense. Hence, we have also examples of semigroups of non-Lebesgue measurable sets (the same families $\mathcal{S}_{\mathcal{V}}$ and $\mathcal{S}_{\mathcal{V}}^{*}$ ) which constitute answers to the question posed above.

Let us note that one can produce other subfamilies of $\mathscr{B}_{p}^{c} \cap \mathcal{L}^{c}$ which are invariant under translations of $\mathbb{R}$ and which are even abelian groups in some natural sense. For that, it is enough to consider the family $\mathcal{F}$ of all cosets (which are Bernstein sets) of a subgroup $G$ mentioned above (the group operation on $\mathcal{F}$ is defined by a natural way).

Moreover, the family $\mathcal{F}_{\text {fin }}$ consisting of all finite unions of elements of $\mathcal{F}$ is an abelian semigroup of sets with respect to the operation of union of sets, it is invariant under translations of $\mathbb{R}$ and $\mathcal{F}_{\text {fin }} \subseteq \mathscr{B}_{p}^{c} \cap \mathcal{L}^{c}$. 
Similarly, the dual family $\mathcal{F}_{\text {fin }}^{*}=\left\{\mathbb{R} \backslash F: F \in \mathcal{F}_{\text {fin }}\right\}$ is also an abelian semigroup of sets with respect to the operation of intersection of sets, it is invariant under translations of $\mathbb{R}$ and $\mathcal{F}_{\text {fin }}^{*} \subseteq \mathscr{B}_{p}^{c} \cap \mathcal{L}^{c}$.

Let us point out some simple difference between elements of $\mathcal{S}_{\mathcal{V}}$ and elements of $\mathcal{F}_{\text {fin }}$. Each element of $\mathcal{F}_{\text {fin }}$ as a Bernstein set must be everywhere dense in $\mathbb{R}$ however each element of $\mathcal{S}_{\mathcal{V}}$ does not need to be everywhere dense in $\mathbb{R}$.

For more details and other results regarding elements of the families $\mathscr{B}_{p}, \mathscr{B}_{p}^{c}, \mathcal{L}$ and $\mathcal{L}^{c}$ we refer to $\mathrm{Ku}, \mathrm{Ox}, \mathrm{Kh} 1$.

\subsection{Aim of the thesis}

The aim of this thesis is to present our contribution in the study of the families of sets with(out) the Baire property, and the family of non-measurable sets in the Lebesgue sense. Inspired by Chatyrko's results [Ch1, Ch2 related to sets with(out) the Baire property on the real line, and Kharazishvili generalization of Vitali sets to Vitali selectors [Kh1], we obtain more extended results on the real line and even in more general topological spaces.

Our contribution can be summarized into the following four points.

Firstly, on the real line $\mathbb{R}$ we consider Vitali selectors related to different countable dense subgroups of the additive group $(\mathbb{R},+)$. Following Chatyrko's method and using subideals of meager sets on $\mathbb{R}$, we produce diverse abelian semigroups with respect to the operation of union of sets. Those semigroups consist of sets without the Baire property, and they are invariant under translations of $\mathbb{R}$. The dual semigroups of sets with respect to the operation of intersection of sets possess the same properties. We then extend these results to Euclidean spaces $\mathbb{R}^{n}, n \geq 2$, and to the products of $\mathbb{R}^{n}$ with finite powers of the Sorgenfrey line. Using the ideal of null sets, we also produce semigroups which consist of non-Lebesgue measurable sets, and which are invariant under translations of $\mathbb{R}$ (see Paper I and Paper III).

Secondly, we study countable families of sets without the Baire property. We consider a topological space $X$, which has an open subset homeomorphic to the space $\left(\mathbb{R}^{n}, \tau\right)$ for some admissible extension $\tau$ of the Euclidean topology on $\mathbb{R}^{n}$, and suggest a way of constructing a countable decomposition $\left\{X_{i}\right\}_{i=1}^{\infty}$ of $X$ such that the union of each non-empty proper subfamily of $\left\{X_{i}\right\}_{i=1}^{\infty}$ does not have the Baire property in $X$ (see Paper II).

Thirdly, we show that Vitali selectors related to an abelian Hausdorff nonmeager without isolated points topological group having countable dense subgroups can be used in the construction of a new topology on the underlying set. We then study properties of the new topological space, and show that any space obtained in this manner is hyperconnected and $T_{1}$, and that any infinite product of such spaces with the box topology is hyperconnected, in particular, connected. Fur- 
thermore, we show that any two spaces obtained in this way are homeomorphic, but that no such a space can be a topological group (see Paper IV).

Fourthly, we consider two topologies $\tau$ and $\sigma$ on the same set $X$, and study conditions on $\tau$ and $\sigma$ that imply a relationship (inclusion or equality) between the family of sets with the Baire property in $(X, \tau)$ and the family of sets with the Baire property in $(X, \sigma)$. In particular, we consider the case in which $\tau$ and $\sigma$ are *-topologies formed by using the local function and ideals of sets. Furthermore, we study the same problem for the families of nowhere dense sets and meager sets respectively (see Paper V).

There are still many open problems to be further investigated. In this thesis we point out a few of them. 



\section{Background}

The purpose of this chapter is to fix notation and terminology, and to present some auxiliary facts from set theory, topological groups and dimension theory that are extensively used in our research papers.

\subsection{Algebraic notions in Set Theory}

In this section, we give a short introduction to families of sets with algebraic properties. By a family of sets we mean any set whose elements are themselves sets. Families of sets are mostly denoted by capital scripts letters like $\mathscr{A}, \mathscr{B}$, and so forth.

Let $X$ be a set and $\mathscr{P}(X)$ be the family of all subsets of $X$.

Definition 2.1. A family $\mathscr{A} \subseteq \mathscr{P}(X)$ of sets is called a $\sigma$-algebra on $X$ if the following conditions are satisfied:

(i) $X \in \mathscr{A}$.

(ii) If $A \in \mathscr{A}$ then $A^{c} \in \mathscr{A}$, where $A^{c}=X \backslash A$ is the complement of $A$ in $X$.

(iii) If $A_{1}, A_{2}, \cdots$ is a countable collection of sets in $\mathscr{A}$ then the union $\bigcup_{i=1}^{\infty} A_{i}$ is also in $\mathscr{A}$.

From the De Morgan's laws, each $\sigma$-algebra is also closed under countable intersections of sets and it contains also the empty set $\emptyset$. 
Definition 2.2. (a) A non-empty family $\mathscr{I} \subseteq \mathscr{P}(X)$ of sets is called an ideal of sets on $X$ if it satisfies the following conditions:

(i) If $A \in \mathscr{I}$ and $B \in \mathscr{I}$ then $A \cup B \in \mathscr{I}$.

(ii) If $A \in \mathscr{I}$ and $B \subseteq A$ then $B \in \mathscr{I}$.

(b) If an ideal of sets $\mathscr{I}$ is closed under countable unions, then it is called a $\sigma$-ideal of sets on $X$.

Example 2.3. If $A$ is a subset of $X$ then the family $\mathscr{I}_{A}$ of all subsets of $A$ forms a $\sigma$-ideal of sets on $X$. The family $\mathscr{I}_{f}$ of all finite subsets of $X$ forms an ideal of sets on $X$, but not a $\sigma$-ideal whenever $X$ is infinite. The family $\mathscr{I}_{c}$ of all countable subsets of $X$ forms a $\sigma$-ideal of sets on $X$.

Given a family $\mathscr{A}$ of subsets of $X$, the smallest $\sigma$-algebra containing $\mathscr{A}$ is called the $\sigma$-algebra generated by $\mathscr{A}$. It is the intersection of all $\sigma$-algebras containing the family $\mathscr{A}$.

Example 2.4. Let $\left(\mathbb{R}, \tau_{E}\right)$ be the real line, i.e., the set of real numbers $\mathbb{R}$ endowed with the topology $\tau_{E}$ defined by all open intervals of $\mathbb{R}$. The $\sigma$-algebra generated by the collection of all open intervals of $\mathbb{R}$ is called the Borel $\sigma$-algebra, and its elements are called the Borel sets. The $\sigma$-algebra generated by the ideal of Lebesgue measure zero sets (null sets) together with the Borel sets equals the collection $\mathcal{L}$ of all Lebesgue measurable subsets of $\mathbb{R}$.

Another type of family of sets which is important in this work is a semigroup of sets.

Definition 2.5. A non-empty family $\mathcal{S} \subseteq \mathscr{P}(X)$ of sets is called a semigroup of sets with respect to the operation of union (resp. intersection) of sets on $X$ if $\mathcal{S}$ is closed under finite unions (resp. intersections) of sets, i.e., if $A_{1}, A_{2}, \cdots, A_{n} \in \mathcal{S}$ then $\bigcup_{i=1}^{n} A_{i} \in \mathcal{S}\left(\operatorname{resp} . \bigcap_{i=1}^{n} A_{i} \in \mathcal{S}\right)$.

Note that if $\mathcal{S}$ is a semigroup of sets with respect to the operation of union of sets then the dual family $\mathcal{S}^{*}=\{X \backslash S: S \in \mathcal{S}\}$ is also a semigroup of sets with respect to the operation of intersection of sets, and vice versa.

In this thesis we will discuss only semigroups of sets with respect to the operation of union of sets (shortly, semigroups of sets).

Example 2.6. Let $\mathscr{A}$ be a non-empty family of subsets of $X$. Define $\mathcal{S}_{\mathscr{A}}=$ $\left\{\bigcup_{i=1}^{n} A_{i}: A_{i} \in \mathscr{A}, n \in \mathbb{N}\right\}$, where $\mathbb{N}$ is the set of positive integers. It is clear that $\mathcal{S}_{\mathscr{A}}$ is a semigroup of sets on $X$. We will call $\mathcal{S}_{\mathscr{A}}$ the semigroup of sets generated by $\mathscr{A}$.

We now present two ways of extending a given semigroup by the use of an ideal of sets. Let $\mathscr{A}$ and $\mathscr{B}$ be families of subsets of $X$. Define $\mathscr{A} \vee \mathscr{B}=\{A \cup B: A \in$ $\mathscr{A}, B \in \mathscr{B}\}$ and $\mathscr{A} * \mathscr{B}=\left\{\left(A \backslash B_{1}\right) \cup B_{2}: A \in \mathscr{A}, B_{1} \in \mathscr{B}, B_{2} \in \mathscr{B}\right\}$. 
Proposition 2.7. Let $\mathcal{S}$ be a semigroup of sets and let $\mathscr{I}$ be an ideal of sets on $X$. Then the families $\mathcal{S} \vee \mathscr{I}$ and $\mathcal{S} * \mathscr{I}$ are semigroups of sets on $X$ such that $\mathcal{S} \subseteq \mathcal{S} \vee \mathscr{I} \subseteq \mathcal{S} * \mathscr{I}$. Moreover, $(\mathcal{S} * \mathscr{I}) * \mathscr{I}=\mathcal{S} * \mathscr{I}$ and $(\mathcal{S} \vee \mathscr{I}) \vee \mathscr{I}=\mathcal{S} \vee \mathscr{I}$

The proof is elementary and it is based on the definition of ideals of sets.

\subsection{Sets with the Baire property}

In this section, we recall some definitions and basic facts related to sets with the Baire property. This property is a classical topological notion which is originated in the thesis of R. Baire.

Below $X$ is assumed to be a non-empty topological space. For a subset $A$ of $X$, the notation $\mathrm{Cl}_{X}(A)$ (resp. $\operatorname{Int}_{X}(A)$ ) stands for the closure (resp. interior) of $A$ in $X$.

Definition 2.8. Let $A$ be a subset of $X$.

The set $A$ is said to be a nowhere dense set in $X$ if its closure has an empty interior, i.e., $\operatorname{Int}_{X} \mathrm{Cl}_{X}(A)=\emptyset$.

The set $A$ is said to be dense in $X$ if the closure of $A$ is $X$, i.e., $\mathrm{Cl}_{X}(A)=X$.

It can be shown that every subset of a nowhere dense set is also a nowhere dense set, and that any finite union of nowhere dense sets is again a nowhere dense set. Hence, the family of all nowhere dense sets in $X$ forms an ideal of sets.

Note that if $A$ is dense in $X$ and $A \subseteq B \subseteq X$ then $B$ is also dense in $X$.

Example 2.9. Let $\mathbb{R}$ be the real line. The set $\mathbb{Z}$ of all integers is a nowhere dense subset of $\mathbb{R}$. However, the set $\mathbb{Q}$ of rational numbers, as well as its extensions $\mathbb{Q}(\alpha)=\{a+b \alpha: a \in \mathbb{Q}, b \in \mathbb{Q}\}$ for each irrational number $\alpha$, is a (countable) dense subset of $\mathbb{R}$.

It is not true in general that a countable union of nowhere dense sets is a nowhere dense set. In fact, the set $\mathbb{Q}$ is a union of countably many nowhere dense sets in $\mathbb{R}$, however $\operatorname{Int}_{\mathbb{R}} \mathrm{Cl}_{\mathbb{R}}(\mathbb{Q})=\mathbb{R}$.

Definition 2.10. A subset $A$ of $X$ is called meager (or of first category) in $X$ if $A$ can be represented as a countable union of nowhere dense sets in $X$. Any set that is not meager in $X$ is called nonmeager (or of second category) in $X$.

The family of all meager subsets of $X$ forms a $\sigma$-ideal of sets. The fundamental theorem of R. Baire asserts the following $[\mathrm{KF}$.

Theorem 2.11 (Baire Category Theorem). Let $X$ be a complete metric space. Then $X$ cannot be covered by countably many nowhere dense subsets of X. Moreover, the union of countably many nowhere dense subsets of $X$ has a dense complement. 
Recall that the real line $\mathbb{R}$ is a complete metric space. Consequently, by the Baire Category Theorem, $\mathbb{R}$ is of second category.

Note that meager sets (resp. sets with Lebesgue measure zero) on $\mathbb{R}$ are small in a topological point of view (resp. in a measure theoretical point of view). Nevertheless, a set that is small in one sense may be large in some other sense.

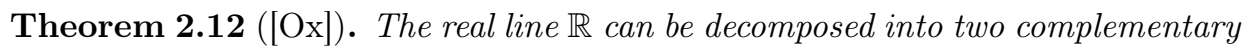
sets $A$ and $B$ such that $A$ is of first category and $B$ is of Lebesgue measure zero.

It follows from Theorem 2.12 that every subset of the real line can be represented as a disjoint union of a null set and a set of first category.

Definition 2.13. A subset $A$ of $X$ is said to have the Baire property in $X$ if it can be represented in the form $A=(O \backslash M) \cup N$, where $O$ is an open set of $X$ and $M, N$ are meager sets in $X$.

Note that a subset $A$ of $X$ has the Baire property in $X$ iff there are an open set $O$ of $X$ and a meager set $M$ of $X$ such that $A=O \Delta M$, where $\Delta$ is the symmetric difference set operation.

The family $\mathscr{B}_{p}(X)$ of sets with the Baire property in $X$ is a $\sigma$-algebra of sets, which is generated by the family $\mathcal{M}$ of all meager sets in $X$ together with the family $\mathcal{O}$ of all open sets in $X$. Furthermore, it is invariant under the action of the group $\mathscr{H}(X)$ of all homeomophisms of $X$, i.e., if $A \in \mathscr{B}_{p}(X)$ and $h \in \mathscr{H}(X)$ then $h(A) \in \mathscr{B}_{p}(X)$. Let us also notice that the family $\mathcal{O}$ is a semigroup of sets on $X$ such that $\mathcal{S}_{\mathcal{O}}=\mathcal{O}$, and that $\mathscr{B}_{p}(X)=\mathcal{O} * \mathcal{M}$.

An important question is to know whether every subset of $X$ possesses the Baire property in $X$. There exist topologies on $X$ such that the family of sets having the Baire property in $X$ contains every subset of $X$, whereas there exist topologies on $X$ such that the family of sets with the Baire property in $X$ does not contain every subset of $X$. In Section 2.7, we will present examples of sets without the Baire property on the real line.

For a more detailed description of sets with the Baire property, we refer to $\mathrm{Ku}, \mathrm{Ox}$.

\subsection{Elements of topological groups}

The results which are presented in the fourth paper partially concern topological groups. Thereupon, in this section, we present some notions and concepts which are related to topological groups.

Definition 2.14. A set $G$ which is endowed with the structures of a(n abelian) group and a topological space is called a(n abelian) topological group if the multiplicative mapping $G \times G \longrightarrow G,(x, y) \longmapsto x \cdot y$, and the inverse mapping 
$G \longrightarrow G, x \longmapsto x^{-1}$ are continuous, where $G \times G$ is viewed as a topological space by using the product topology.

Note that we have used a multiplicative group notation for the group operation.

We give some examples of abelian topological groups. The real numbers $\mathbb{R}$ with the addition $x+y: x, y \in \mathbb{R}$ as the group operation endowed with the standard topology, the non-zero real numbers $\mathbb{R} \backslash\{0\}$ with the product $x \cdot y: x, y \in \mathbb{R}$ as the group operation endowed with the subspace topology from the standard topology on $\mathbb{R}$, the set $\mathbb{T}$ of complex numbers of modulus one with the product $z_{1} \cdot z_{2}: z_{1}, z_{2} \in \mathbb{T}$ as the group operation endowed with the subspace topology from the complex plane, finite products $\mathbb{R}^{n}, \mathbb{T}^{n}$ and the products $\mathbb{R}^{n} \times \mathbb{T}^{m}, n \geq 1, m \geq 1$, where the group operations and topologies are defined naturally.

Recall that a topological space $X$ is called:

a $T_{0}$-space if for any two distinct points of $X$, there is an open set which contains one point but not the other;

a $T_{1}$-space if each point of $X$ is a closed set;

a Hausdorff space if for any two distinct points $p, q$ of $X$ there are disjoint open sets $U, V$ such that $p \in U$ and $q \in V$;

a regular $T_{1}$-space if $X$ is $T_{1}$ and for any point $p$ and any closed set $F$ such that $p \notin F$ there are disjoint open sets $U, V$ such that $p \in U$ and $F \subseteq V$;

a Tychonoff space if $X$ is a $T_{1}$-space and for each closed set $F \subseteq X$ and each point $x \in X \backslash F$, there exists a continuous function $h: X \longrightarrow[0,1]$ such that $h(F)=0$ and $h(x)=1$;

a normal Hausdorff space if $X$ is Hausdorff and for any disjoint closed sets $F$ and $G$ there exist disjoint open sets $U, V$ such that $F \subseteq U$ and $G \subseteq V$.

Let us recall that each normal Hausdorff (resp. Tychonoff, regular $T_{1}$, Hausdorff or $T_{1}$ ) space is Tychonoff (resp. regular $T_{1}$, Hausdorff, $T_{1}$ or $T_{0}$ ). The existence of a compatible group structure on a topological space guarantees good topological properties of the space.

Proposition 2.15 ([ $\mathrm{HR}])$. Let $G$ be a topological group. If $G$ is a $T_{0}$-space then $G$ is Tychonoff.

Let us note that not every topological group is normal Hausdorff. For instance, the group $\mathbb{Z}^{m}$, where $m$ is any uncountable cardinal number, is a Tychonoff space but it is not normal $[\mathrm{HR}$. However, if our group is additionally locally compact the situation will be changed.

Proposition 2.16 ([प] $]$ ). Every locally compact $T_{0}$-topological group is normal Hausdorff.

Let us note that not every topology on a group makes it into a topological group. For example, let $G$ be an arbitrary infinite group endowed with the topol- 
ogy $\tau=\{U \subseteq G: U=\emptyset$ or $G \backslash U$ is finite $\}$. It can be shown that $\tau$ is the weakest possible topology on $G$ which makes $G$ into a $T_{1}$-space. However, $G$ is not a topological group. In fact, $G$ satisfies the $T_{0}$-separation axiom but $G$ is not Hausdorff.

For other notions and facts, we refer the reader to $[\mathrm{HR}, \mathrm{Mo}]$.

\subsection{Topological products of an infinite family of spaces}

Let $X_{\alpha}, \alpha \in I$, be a nondegenerated topological space for each $\alpha \in I$ and $I$ infinite. Then

the product topology on the product $\prod_{\alpha \in I} X_{\alpha}$, is that topology which has a basis for its open sets the collection of all sets of the form $\prod_{\alpha \in I} U_{\alpha}$, where each $U_{\alpha}$ is open in $X_{\alpha}$, and $U_{\alpha} \neq X_{\alpha}$ for only finitely many $\alpha$;

the box product topology on the product $\prod_{\alpha \in I} X_{\alpha}$ is that topology which has a basis for its open sets the collection of all sets of the form $\prod_{\alpha \in I} U_{\alpha}$, where $U_{\alpha}$ is open in $G_{\alpha}$ for each $\alpha \in I$.

It is well known that if each topological space $X_{\alpha}, \alpha \in I$, is connected then $\prod_{\alpha \in I} X_{\alpha}$ with the product topology is also connected as a topological space.

However, $\prod_{\alpha \in I} X_{\alpha}$ with the box product topology is disconnected as a topological space whenever each $X_{\alpha}$ is regular $T_{1}$. If a similar statement holds for Hausdorff spaces it is unknown.

Let us note that there are examples of $\prod_{\alpha \in I} X_{\alpha}$ with the box product topology which are connected. In the fourth paper we present such products where all spaces are $T_{1}$.

\subsection{Extension of topologies via ideals}

In this section, we describe a method which is used to extend topologies by using ideals of sets. The method was introduced by Janković and Hamlett in their joint work $[\mathrm{JH}]$. Many results which are presented in the fifth paper are based on topologies which are constructed by the use of this method.

Let $(X, \tau)$ be a topological space, $\mathscr{I}$ an ideal of sets on $X$ and let $A$ be a subset of $X$. For each element $x$ of $X$ denote by $\mathscr{N}(x)=\{U \in \tau: x \in U\}$ the family of all open neighborhoods at the point $x$.

Definition $2.17([\mathbf{H a}])$. The set $A^{*}(\mathscr{I})=\{x \in X: A \cap U \notin \mathscr{I}$ for every $U \in$ $\mathscr{N}(x)\}$ is called the local function of $A$ with respect to the ideal of sets $\mathscr{I}$ and the topology $\tau$. 
The following theorem establishes some basic properties of the local function.

Theorem $2.18([\overline{\mathrm{JH}}])$. Let $(X, \tau)$ be a topological space, $\mathscr{I}$ and $\mathscr{J}$ be ideals of sets on $X$ and let $A$ and $B$ be subsets of $X$. The following statements hold:
(i) If $A \subseteq B$ then $A^{*}(\mathscr{I}) \subseteq B^{*}(\mathscr{I})$.
(iv) $\left[A^{*}(\mathscr{I})\right]^{*}(\mathscr{I}) \subseteq A^{*}(\mathscr{I})$.
(ii) $(A \cup B)^{*}(\mathscr{I})=A^{*}(\mathscr{I}) \cup B^{*}(\mathscr{I})$.
(v) If $I \in \mathscr{I}$ then $(A \cup I)^{*}(\mathscr{I})=$ $(A \backslash I)^{*}(\mathscr{I})=A^{*}(\mathscr{I})$.
(iii) $A^{*}(\mathscr{I})=C l\left(A^{*}(\mathscr{I})\right) \subseteq C l(A)$.
(vi) If $\mathscr{I} \subseteq \mathscr{J}$ then $A^{*}(\mathscr{J}) \subseteq A^{*}(\mathscr{I})$.

Recall that a point $x \in X$ is said to be an $\omega$-accumulation (resp. a condensation) point of $A \subseteq X$ if every neighborhood of $x$ contains an infinite (resp. uncountable) number of points of $A$.

Example 2.19. We present some examples of local functions: If $\mathscr{I}=\{\emptyset\}$ then $A^{*}(\mathscr{I})=\mathrm{Cl}(A)$. If $\mathscr{I}=\mathscr{P}(X)$ then $A^{*}(\mathscr{I})=\emptyset$. If $\mathscr{I}=\mathscr{I}_{f}$ then $A^{*}(\mathscr{I})$ is the set of all $\omega$-accumulation points of $A$. If $\mathscr{I}=\mathscr{I}_{c}$ then $A^{*}(\mathscr{I})$ is the set of all condensation points of $A$. If $A$ is an element of $\mathscr{I}$ then $A^{*}(\mathscr{I})=\emptyset$ for every ideal of sets $\mathscr{I}$ on $X$.

It is well known $[\mathrm{Ku}$ ] that if $\mathrm{Cl}$ is a Kuratowski closure operator, i.e., $\mathrm{Cl}$ is an operator from $\mathscr{P}(X)$ to $\mathscr{P}(X)$ such that $\mathrm{Cl}(\emptyset)=\emptyset, A \subseteq \mathrm{Cl}(A), \operatorname{Cl}(A \cup B)=$ $\mathrm{Cl}(A) \cup \mathrm{Cl}(B)$ and $\mathrm{Cl}(\mathrm{Cl}(A))=\mathrm{Cl}(A)$ for all $A, B \subseteq X$, then there exists a unique topology on $X$ whose closed sets are given by the collection $\{F \subseteq X: \mathrm{Cl}(F)=F\}$.

By Theorem 2.18, the operator $\mathrm{Cl}^{*}($.$) from \mathscr{P}(X)$ to $\mathscr{P}(X)$ defined by $\mathrm{Cl}^{*}(A)=$ $A \cup A^{*}(\mathscr{I})$ for every subset $A$ of $X$, is a Kuratowski closure operator. Hence the family $\left\{U \subseteq X: \mathrm{Cl}^{*}(X \backslash U)=X \backslash U\right\}$ is a topology on $X$, which is denoted by $\tau^{*}(\mathscr{I})$. The topology $\tau^{*}(\mathscr{I})$ is called the $*$-topology (or new topology) from the old topology $\tau$ and the ideal of sets $\mathscr{I}$. It has the collection $\beta(\tau, \mathscr{I})=\{O \backslash I: O \in \tau, I \in \mathscr{I}\}$ as a base. Hence $\tau \subseteq \tau^{*}(\mathscr{I})$. One of the basic properties of the $*$-topologies is that each element $I \in \mathscr{I}$ is closed and discrete in $\left(X, \tau^{*}(\mathscr{I})\right)$.

By Example 2.19 it follows that if $\mathscr{I}=\{\emptyset\}$ then $\mathrm{Cl}^{*}(A)=\mathrm{Cl}(A)$, and hence $\tau^{*}(\mathscr{I})=\tau$. Furthermore, if $\mathscr{I}=\mathscr{P}(X)$ then $\mathrm{Cl}^{*}(A)=A$, and hence $\tau^{*}(\mathscr{I})=$ $\tau_{\text {disc }}$, where $\tau_{\text {disc }}$ is the discrete topology on $X$.

It follows from the Theorem 2.18 (vi) that if $\mathscr{I}_{1}$ and $\mathscr{I}_{2}$ are ideals of sets on $X$ such that $\mathscr{I}_{1} \subseteq \mathscr{I}_{2}$ and $A \subseteq X$ is closed in $\tau^{*}\left(\mathscr{I}_{1}\right)$ then $A$ is also closed in $\tau^{*}\left(\mathscr{I}_{2}\right)$, i.e., $\tau^{*}\left(\mathscr{I}_{1}\right) \subseteq \tau^{*}\left(\mathscr{I}_{2}\right)$. Hence, $\tau \subseteq \tau^{*}(\mathscr{I}) \subseteq \tau_{\text {disc }}$ for every ideal $\mathscr{I}$ of sets on $X$. Moreover, there exist ideals of sets $\mathscr{I}$ different from $\mathscr{I}=\{\emptyset\}$ such that $\tau^{*}(\mathscr{I})=\tau$ and there exist ideals $\mathscr{I}$ of sets on $X$ different from $\mathscr{P}(X)$ such that $\tau^{*}(\mathscr{I})=\tau_{\text {disc }}$.

For other notions and facts, we refer the reader to $[\mathrm{Ku}, \mathrm{JH}]$. 


\subsection{Admissible extensions of topologies}

Given two different topologies $\tau$ and $\sigma$ on the same underlying set $X$, one can be interested in the study of conditions on $\tau$ and $\sigma$ that imply some relationship (for example inclusion or equality) between the family of sets with the Baire property in $(X, \tau)$ and the family of sets with the Baire property in $(X, \sigma)$.

Definition 2.20 ([CN]). Let $\tau$ and $\sigma$ be topologies on a set $X$. The topology $\sigma$ is said to be an admissible extension of the topology $\tau$ on $X$ if

(i) $\tau \subseteq \sigma$, and

(ii) $\tau$ is a $\pi$-base of $\sigma$, i.e., for each non-empty element $O$ of $\sigma$ there is a nonempty element $V$ in $\tau$ such that $V \subseteq O$.

Now we explain why the concept of admissible extension is important.

Theorem 2.21. Let $X$ be a set, $\tau$ and $\sigma$ be topologies on $X$ such that $\sigma$ is an admissible extension of $\tau$. Then the spaces $(X, \tau)$ and $(X, \sigma)$ have the same families of nowhere dense sets (resp. meager sets or sets with the Baire property).

Proof. Let $N$ be a nowhere dense set in the space $(X, \tau)$. We will show that $N$ is also a nowhere dense set in the space $(X, \sigma)$. Consider a non-empty set $O_{\sigma} \in \sigma$. Since $\sigma$ is an admissible extension of $\tau$, there exists a non-empty set $O_{\tau} \in \tau$ such that $O_{\tau} \subseteq O_{\sigma}$. Since $N$ is a nowhere dense set in the space $(X, \tau)$, there is a non-empty set $V_{\tau} \in \tau$ such that $V_{\tau} \subseteq O_{\tau}$ and $V_{\tau} \cap N=\emptyset$. Note that $V_{\tau} \in \sigma$ and $V_{\tau} \subseteq O_{\sigma}$. Hence we get that $N$ is a nowhere dense set in the space $(X, \sigma)$. The inverse implication can be proved by a similar simple argument. The case of meager sets is obvious.

Since $\tau \subseteq \sigma$, it is clear that each subset $A$ of $X$ with the Baire property in $(X, \tau)$ also has the Baire property in $(X, \sigma)$. We refer to $\mathrm{ACN}$ for the other inclusion.

Example 2.22 (Sorgenfrey line). The first example of an admissible extension of the Euclidean topology $\tau_{E}$ is the Sorgenfrey topology $\tau_{S}$ on $\mathbb{R}$. Recall that the base of $\tau_{S}$ is given by the collection $\{[a, b): a, b \in \mathbb{R}, a<b\}$ and the topological space $\left(\mathbb{R}, \tau_{S}\right)$ is called the Sorgenfrey line. In general, for each integer $k \geq 1$ and each integer $m \geq 0$ the topology $\tau_{S}^{k} \times \tau_{E}^{m}$ on $\mathbb{R}^{k+m}$, where $\tau_{S}^{k}=\prod_{j=1}^{k}\left(\tau_{S}\right)_{j}$ and $\left(\tau_{S}\right)_{j}=\tau_{S}$ for each $j \leq k$, is an admissible extension of $\tau_{E}^{k+m}$ on $\mathbb{R}^{k+m}$.

More general examples of admissible extensions of the Euclidean topology $\tau_{E}$ on $\mathbb{R}$ can be found in $[\mathrm{CH}]$.

Example 2.23 (Hattori spaces). Let $A$ be a subset of $\mathbb{R}$. In his work [Ht], Hattori defined a topology $\tau(A)$ on $\mathbb{R}$ in the following way: 
(i) for each $x \in A,\{(x-\varepsilon, x+\varepsilon): \varepsilon>0\}$ is the neighborhood base at $x$.

(ii) for each $x \in \mathbb{R} \backslash A,\{[x, x+\varepsilon): \varepsilon>0\}$ is the neighborhood base at $x$.

In their joint work $\mathrm{CH}$, Chatyrko and Hattori continued to study the topological spaces $(\mathbb{R}, \tau(A)), A \subseteq \mathbb{R}$. They observed that for any subsets $A, B$ of $\mathbb{R}$, $A \supseteq B$ iff $\tau(A) \subseteq \tau(B)$, in particular, $\tau(\mathbb{R})=\tau_{E} \subseteq \tau(A)$ and $\tau(B) \subseteq \tau(\emptyset)=\tau_{S}$. Furthermore, they showed that for every $A \subseteq \mathbb{R}$ the topology $\tau(A)$ is an admissible extension of $\tau_{E}$ on $\mathbb{R}$. Hence the collection $\{\tau(A): A \subseteq \mathbb{R}\}$ is a poset of topologies on $\mathbb{R}$, which consists of admissible extensions of the topology $\tau_{E}$ on $\mathbb{R}$. The topological spaces $(\mathbb{R}, \tau(A)), A \subseteq \mathbb{R}$ are known as Hattori spaces. All the spaces $(\mathbb{R}, \tau(A)), A \subseteq \mathbb{R}$ are regular hereditarily Lindelöf and hereditarily separable.

Other examples of admissible extensions of the topology $\tau_{E}$ on $\mathbb{R}$, for which the construction is different from the previous ones, can be obtained by using the family of nowhere dense sets on the real line.

Example 2.24 (Admissible extensions via nowhere dense sets). Consider a subideal $\mathscr{I}$ of the family of nowhere dense sets of the real line. The topology $\tau_{E}^{*}(\mathscr{I})$ is an admissible extension of $\tau_{E}$ on $\mathbb{R}$. In fact, since $\tau_{E} \subseteq \tau_{E}^{*}(\mathscr{I})$, it is enough to show that $\tau_{E}$ is a $\pi$-base of $\tau_{E}^{*}(\mathscr{I})$. Let $O$ be a non-empty element of $\tau_{E}^{*}(\mathscr{I})$. We can assume that $O=U \backslash I$ where $\emptyset \neq U \in \tau_{E}$ and $I \in \mathscr{I}$. Since $I$ is a nowhere dense set in $\left(\mathbb{R}, \tau_{E}\right)$, there exists a non-empty element $V$ of $\tau_{E}$ such that $V \subseteq U$ and $V \cap I=\emptyset$. It is clear that $V \subseteq O$.

Note that each topology $\tau(A), A \subsetneq \mathbb{R}$ from the Example 2.23 cannot be a $*-$ topology from the Euclidean topology on the reals. Hence the topologies described in Example 2.23 are different from those described in the Example 2.24.

\subsection{Vitali selectors on the real line}

In this section, we present two types of sets which cannot be Lebesgue measurable and which cannot have the Baire property on the real line.

The first type of such sets is called Vitali selectors. The Vitali selectors are closely related to Vitali sets which were introduced by G. Vitali in 1905.

Let $Q$ be a countable dense subgroup of the additive group $(\mathbb{R},+)$. For an element $x \in \mathbb{R}$, denote by $T_{x}$ the translation of $\mathbb{R}$ by $x$, i.e., $T_{x}(y)=y+x$ for each element $y \in \mathbb{R}$. If $A$ is a subset of $\mathbb{R}$ and $x \in \mathbb{R}$, denote the set $T_{x}(A)=$ $\{a+x: a \in A\}$ by $A_{x}$. Define the equivalence relation $E$ on $\mathbb{R}$ as follows: for $x, y \in \mathbb{R}$, let $x E y$ if and only if $x-y \in Q$, and let $\left\{E_{\alpha}(Q): \alpha \in I\right\}$ be the set of all equivalence classes, where $I$ is some indexing set. Observe that the cardinality $|I|$ of $I$ is equal to $\mathfrak{c}$ (the continuum), and that for each $\alpha \in I$ and each $x \in E_{\alpha}(Q)$ we have $E_{\alpha}(Q)=Q+x$. Therefore, each equivalence class $E_{\alpha}(Q)$ is dense in $\mathbb{R}$. 
Definition 2.25 ([Kh1] ). A Vitali $Q$-selector of $\mathbb{R}$ is any subset $V$ of $\mathbb{R}$ such that $\left|V \cap E_{\alpha}(Q)\right|=1$ for each $\alpha \in I$.

A Vitali $Q$-selector is called a Vitali set $[\mathrm{Vi}]$ whenever the $\operatorname{subgroup} Q$ coincides with the group $\mathbb{Q}$ of rational numbers.

For Vitali selectors, the following statements hold.

Proposition 2.26. Let $V$ be a Vitali $Q$-selector of $\mathbb{R}$. Then we have the following.

(i) If $q_{1}, q_{2} \in Q$ and $q_{1} \neq q_{2}$ then $\left(V+q_{1}\right) \cap\left(V+q_{2}\right)=\emptyset$.

(ii) $\mathbb{R}=\bigcup_{q \in Q}(V+q)$.

(iii) The set $V$ is not meager in $\mathbb{R}$.

(iv) The set $V+x$ is also a Vitali $Q$-selector for each $x \in \mathbb{R}$.

Let us point out that there exist Vitali $Q$-selectors of the real line which have some additional properties. In fact, there exist bounded Vitali $Q$-selectors and there exist Vitali $Q$-selectors which are dense in any non-empty open subsets of $\mathbb{R}$ (see Ch2, Propositions 3.1 and 3.3).

Theorem 2.27 ([Kh1]). Any Vitali $Q$-selector of $\mathbb{R}$ is non-Lebesgue measurable and does not have the Baire property on the real line.

Let us note that Theorem 2.27 is a particular case of the following more general result; which is valid also for Vitali selectors of the real line.

Theorem 2.28 (Ch1). Let $V_{i}$ be a Vitali set for each $i \leq n$ where $n$ is some integer such that $n \geq 1$. Then the set $U=\bigcup_{i=1}^{n} V_{i}$ does not contain the difference $O \backslash M$, where $O$ is a non-empty open set and $M$ is meager. In particular, the set $U$ does not possess the Baire property on the real line.

A similar result to Theorem 2.28 about non-Lebesgue measurability of finite unions of Vitali sets was proved by Kharazishvili. For a proof, we refer to [Kh2].

Let us notice that in the light of Proposition 2.26 (ii), the results of Theorem 2.28 cannot be extended to all countable union of Vitali sets. However, Chatyrko proved the following statement which is valid not only for Vitali sets but also for Vitali selectors of $\mathbb{R}$.

Theorem 2.29 ([Ch2]). If $V$ is a Vitali set and $\Gamma$ is a non-empty proper subset of $\mathbb{Q}$ then the set $\bigcup_{q \in \Gamma}(V+q)$ does not possess the Baire property on the real line.

The second type of sets which neither possess the Baire property on the real line, nor are measurable in the Lebesgue sense, is called Bernstein sets. Bernstein sets were constructed by F. Bernstein in 1908 and they are defined as follows. 
Definition 2.30 (cf. $[\mathbf{O x}]$ ). A subset $B$ of $\mathbb{R}$ is called a Bernstein set if $F \cap B \neq \emptyset$ and $F \cap(\mathbb{R} \backslash B) \neq \emptyset$ for every uncountable closed subset $F$ of $\mathbb{R}$.

This definition shows directly that the complement $\mathbb{R} \backslash B$ of a Bernstein set $B$ in $\mathbb{R}$ is also a Bernstein set. The construction of Bernstein sets is based on the method of transfinite induction. For the construction of Bernstein sets, we refer to $\mathrm{Ox}, \mathrm{Kh} 1$.

Theorem 2.31 (cf. $[\mathrm{Ox}]$ ). Any Bernstein set B is non-measurable in the Lebesgue sense and does not have the Baire property. Indeed, every Lebesgue measurable subset of either $B$ or $\mathbb{R} \backslash B$ is a null set, and any subset of $B$ or $\mathbb{R} \backslash B$ that has the Baire property is of first category.

Remark 2.32. Apart from Vitali selectors and Bernstein sets, there are other types of sets which are neither Lebesgue measurable, nor have the Baire property on the real line. For instance, non-Lebesgue measurable sets (or sets without the Baire property) associated with Hamel bases. Moreover (see for instance [Kh1]), there exist non-Lebesgue measurable sets (or sets without the Baire property) which have algebraic structure of subgroups of the additive group $(\mathbb{R},+)$.

In a similar way, we can construct Vitali selectors in higher-dimensional Euclidean spaces $\mathbb{R}^{n}$. However, for $n \geq 2$, there exists a subclass of Vitali selectors that we called rectangular Vitali selectors in [N]. These are Vitali selectors which are related to countable dense subgroups of $\left(\mathbb{R}^{n},+\right)$ that are assumed to be product of $n$ countable dense subgroups of $(\mathbb{R},+)$. Hence, each rectangular Vitali selector of $\mathbb{R}^{n}$ is a product of $n$ Vitali selectors of $\mathbb{R}$.

\subsection{Lebesgue covering dimension}

In this section, we review some basic properties of the Lebesgue covering dimension dim in separable metrizable spaces.

Let $X$ be a topological space and let $\mathscr{A}=\left\{A_{\alpha}\right\}_{\alpha \in \Gamma}$ be a family of subsets, not all empty, of $X$, where $\Gamma$ is some indexing set.

The order of the family $\mathscr{A}=\left\{A_{\alpha}\right\}_{\alpha \in \Gamma}$ is the largest integer $n$ for which there exists a subset $I$ of $\Gamma$ with $n+1$ elements such that $\bigcap_{\alpha \in I} A_{\alpha}$ is non-empty, or $\infty$ if there is no such largest integer.

The family $\mathscr{A}=\left\{A_{\alpha}\right\}_{\alpha \in \Gamma}$ is said to be a cover of $X$ if $\bigcup_{\alpha \in \Gamma} A_{\alpha}=X$.

A cover $\mathscr{B}$ is a refinement of another cover $\mathscr{A}$ of the same space $X$ (in other words $\mathscr{B}$ refines $\mathscr{A}$ ), if for every $B \in \mathscr{B}$ there exists an $A \in \mathscr{A}$ such that $B \subseteq A$.

Definition 2.33. Let $X$ be a topological space. Then

- $\operatorname{dim} X=-1$ if and only if $X=\emptyset$. 
- $\operatorname{dim} X \leq n$, where $n=-1,0,1, \cdots$, if every finite open cover of the space $X$ has a finite open refinement of order not exceeding $n$.

- $\operatorname{dim} X=n$ if it is true that $\operatorname{dim} X \leq n$ but it is not true that $\operatorname{dim} X \leq n-1$.

- $\operatorname{dim} X=\infty$ if for every integer $n$ it is false that $\operatorname{dim} X \leq n$ for $n=-1,0,1, \cdots$.

If $\operatorname{dim} X=n$, then $X$ is called an $n$-dimensional topological space. Let us remind that a topological space $X$ is said to be separable if it contains a countable dense subset. The space $X$ is said to be metrizable if there exists a metric on $X$ which induces the topology on $X$.

For separable metrizable spaces, some basic properties of the Lebesgue covering dimension are summarized in the following theorems [En].

Theorem 2.34 (Fundamental Theorem of Dimension). For every natural number $n$, we have $\operatorname{dim} \mathbb{R}^{n}=n$.

Theorem 2.35 (Subspace Theorem). If $Y$ is a subspace of a separable metrizable space $X$, then $\operatorname{dim} Y \leq \operatorname{dim} X$.

Theorem 2.36 (Countable Sum Theorem). Let $X$ be a separable metrizable space and $X=\bigcup_{i=1}^{\infty} F_{i}$, where $F_{i}$ is closed in $X$ for each $i$. If $\operatorname{dim} F_{i} \leq n$ for each $i$, then $\operatorname{dim} X \leq n$.

Theorem 2.37 (Decomposition Theorem). A separable metrizable space $X$ satisfies the inequality $\operatorname{dim} X \leq n$ if and only if $X$ can be represented as the union of $n+1$ subspaces $Z_{1}, Z_{2}, \cdots, Z_{n+1}$ such that $\operatorname{dim} Z_{i} \leq 0$ for $i=1,2, \cdots, n+1$.

Theorem 2.38 (Brouwer Dimension Theorem). Let $Y$ be a subset of $\mathbb{R}^{n}, n \in \mathbb{N}$. Then $\operatorname{dim} Y=n$ if and only if $\operatorname{Int}_{\mathbb{R}^{n}}(Y) \neq \emptyset$.

Theorem 2.39 (Product Theorem). For every pair $X, Y$ of separable metrizable spaces, not both empty, we have $\operatorname{dim}(X \times Y) \leq \operatorname{dim} X+\operatorname{dim} Y$.

Apart from the Lebesgue covering dimension, there exist other dimension functions; for instance the small inductive dimension ind and the large inductive dimension Ind. The three dimensions coincide in the class of separable metrizable spaces, i.e., ind $X=\operatorname{Ind} X=\operatorname{dim} X$ for every separable metrizable space $X$. However, in general spaces, the three functions ind, Ind and dim do not need to coincide.

We refer to [En] for other facts related to the three dimension functions. 


\section{Summary of main results}

\section{Paper I: On the families of sets without the Baire property generated by the Vitali sets}

It is well known that the family of sets with the Baire property on the real line is invariant under translations of $\mathbb{R}$, and that it is closed under all basic set operations. On the other hand, the family of sets without the Baire property is also invariant under translations of $\mathbb{R}$ but it is not closed under basic set operations.

One can pose the following question: Do there exist rich families of subsets of $\mathbb{R}$ without the Baire property, which are invariant under the action of an infinite subgroup of the group $\mathscr{H}(\mathbb{R})$ of all homeomorphisms of $\mathbb{R}$, and on which we can define some algebraic structure from the set-theoretic point of view?

In this paper, we present a positive answer to this question, not only on the real line, but also in higher-dimensional Euclidean spaces $\mathbb{R}^{n}, n \geq 2$. Furthermore, similar results are derived in the case where $\mathbb{R}^{n}$ is endowed with some admissible extensions of the Euclidean topology.

On the real line $\mathbb{R}$, we define the family $\mathcal{V}_{2}=\left\{(U \backslash M) \cup N: U \in \mathcal{V}_{1} ; M, N \in \mathcal{A}\right\}$, where $\mathcal{V}_{1}$ is the family of all finite unions of all Vitali sets of $\mathbb{R}$, and $\mathcal{A}$ is the $\sigma$-ideal of meager subsets of $\mathbb{R}$. We prove that the family $\mathcal{V}_{2}$ consists of zero-dimensional sets without the Baire property, that it is invariant under translations of $\mathbb{R}$, and that it is an abelian semigroup of sets with respect to the operation of union of sets.

For the Euclidean spaces $\mathbb{R}^{n}, n \geq 2$, we define a Vitali set of $\mathbb{R}^{n}$ as a product $\prod_{i=1}^{n} V_{i}$ of Vitali sets $V_{i}, i=1,2, \cdots, n$ of the real line $\mathbb{R}$ (see [N] for a general 
case of Vitali sets), and put $\mathcal{V}_{2}^{n}=\left\{(U \backslash M) \cup N: U \in \mathcal{V}_{1}^{n} ; M, N \in \mathcal{A}^{n}\right\}$, where $\mathcal{V}_{1}^{n}$ is the family of all finite union of all Vitali sets of $\mathbb{R}^{n}$, and $\mathcal{A}^{n}$ is the $\sigma$-ideal of all meager sets on $\mathbb{R}^{n}$. We prove that the family $\mathcal{V}_{2}^{n}$ consists of sets without the Baire property in $\mathbb{R}^{n}$, that it is invariant under translations of $\mathbb{R}^{n}$, and that it is an abelian semigroup of sets with respect to the operation of union of sets. We also prove that $0 \leq \operatorname{dim} A \leq n-1$ for each element $A$ of $\mathcal{V}_{2}^{n}$.

Finally, we introduce a notion of an admissible extension of a topology $\tau_{1}$ on a set $X$ as a new larger than $\tau_{1}$ topology $\tau_{2}$ with some peculiar property on the same set $X$. With the help of the notion we extend the mentioned above results from the Euclidean spaces $\mathbb{R}^{n}$ to products of $\mathbb{R}^{n}$ with finite powers of the Sorgenfrey line.

\section{Paper II: On countable families of sets without the Baire property}

It is well known that any Vitali set does not possess the Baire property on the real line. The same result is valid for finite unions of Vitali sets. It was proved by Chatyrko Ch2 that if $V$ is a Vitali set and $\Gamma$ is a non-empty proper subset of the set $\mathbb{Q}$ of rational numbers, then the set $\bigcup_{q \in \Gamma}(V+q)$ does not possess the Baire property on the real line. He also extended the result to higher-dimensional Euclidean spaces $\mathbb{R}^{n}$ for $n \geq 2$.

In this paper, we generalize Chatyrko's result by replacing Vitali sets by Vitali selectors, and by changing the Euclidean spaces to the spaces $\left(\mathbb{R}^{n}, \tau\right)$ for some admissible extension $\tau$ of the Euclidean topology on $\mathbb{R}^{n}$, where $n$ is some positive integer.

Let $Q$ be a countable dense subgroup of the additive group $\left(\mathbb{R}^{n},+\right)$. We prove that if $V$ is a Vitali $Q$-selector of $\mathbb{R}^{n}$ (see the description of Vitali sets in $\mathbb{R}^{n}$, Paper I) and $\Gamma$ is any non-empty proper subset of $Q$ then the set $\bigcup_{q \in \Gamma}(V+q)$ does not possess the Baire property in $\left(\mathbb{R}^{n}, \tau\right)$, where $\tau$ is any admissible extension of the Euclidean topology on $\mathbb{R}^{n}$.

Furthermore, let $X$ be a topological space which has an open subset homeomorphic to the space $\left(\mathbb{R}^{n}, \tau\right)$ for some admissible extension $\tau$ of the Euclidean topology on $\mathbb{R}^{n}$. We prove that there exists an infinite disjoint countable family $\left\{X_{i}\right\}_{i=1}^{\infty}$ of sets in $X$ such that $\bigcup_{i=1}^{\infty} X_{i}=X$, and such that the union of each non-empty proper subfamily of $\left\{X_{i}\right\}_{i=1}^{\infty}$ does not have the Baire property in $X$. In case $X$ is a separable metrizable finite-dimensional manifold, we prove that each element of the family $\left\{X_{i}\right\}_{i=1}^{\infty}$ can be chosen dense and zero-dimensional.

\section{Paper III: The Algebra of semigroups of sets}

In the first paper, we constructed an abelian semigroup consisting of sets without the Baire property on the real line. In this paper, we continue the study by looking for other families which consist of sets without the Baire property, which 
are invariant under translations of $\mathbb{R}$, and which are semigroups of sets with respect to the operation of union of sets.

We develop a theory of semigroups with respect to the operation of union of sets and ideals of sets. By applying this theory to Vitali selectors of $\mathbb{R}$, we obtain diverse abelian semigroups which consist of sets without the Baire property, and which are invariant under translations of $\mathbb{R}$. In particular, many results from the first paper can be proven within this theory.

So first, we consider any countable dense subgroup $Q$ of the additive group $(\mathbb{R},+)$, and the family $\mathcal{V}_{1}(Q)$ of all finite unions of Vitali $Q$-selectors of $\mathbb{R}$ (analogues of Vitali sets of $\mathbb{R}$ ). We note that the family $\mathcal{V}_{1}(Q)$ is an abelian semigroup which consists of sets without the Baire property in $\mathbb{R}$, and that it is invariant under translations of $\mathbb{R}$.

Next, we consider the collection $\left\{\mathcal{V}_{1}(Q): Q\right.$ is a countable dense subgroup of $(\mathbb{R},+)\}$ of those semigroups, and study the relationship (in the sense of inclusion) between members of this collection. In particular, we prove that there is no semigroup $\mathcal{V}_{1}(Q)$ which contains all others. This leads us to define a semigroup $\mathcal{V}_{1}^{\text {sup }}$ based on all Vitali selectors which we call a supersemigroup of Vitali selectors. Naturally, the supersemigroup $\mathcal{V}_{1}^{\text {sup }}$ contains all semigroups $\mathcal{V}_{1}(Q)$, where $Q$ is any countable dense subgroup of $(\mathbb{R},+)$. We prove that the supersemigroup $\mathcal{V}_{1}^{\text {sup }}$ consists of sets without the Baire property in $\mathbb{R}$, and that it is invariant under translations of $\mathbb{R}$.

Finally, all the above mentioned semigroups are enlarged with the help of meager sets on $\mathbb{R}$ to semigroups whose elements are of the form $(U \backslash M) \cup N$, where $U \in \mathcal{V}_{1}(Q)$ and $M, N$ are meager subsets of $\mathbb{R}$. We then generalize the construction to finite-dimensional Euclidean spaces $\mathbb{R}^{n}$ for $n \geq 2$. Note that a more extended description can be found in $[\mathrm{N}]$.

\section{Paper IV: Vitali selectors in topological groups and related semigroups of sets}

Let $G$ be an abelian Hausdorff nonmeager without isolated points topological group having countable dense subgroups. In this paper, we use Vitali selectors of $G$ to define a new topology on $G$, and then study its topological properties.

Let $Q$ be a countable dense subgroup of $G$, and let $\mathcal{V}_{1}(Q)$ be the family of all finite unions of Vitali selectors of $G$ related to the subgroup $Q$. We prove that the collection $\left\{G \backslash U: U \in \mathcal{V}_{1}(Q)\right\}$ is a base for a topology $\tau(Q)$ on $G$, which makes $G$ into a new topological space that is denoted by $G(Q)$.

Furthermore, we study the topological properties of the space $G(Q)$. We prove that $G(Q)$ is a $T_{1}$-space but it is not Hausdorff (and hence it cannot be a topological group), and that it is hyperconnected. As a consequence, any infinite product of the spaces $G(Q)$, where $Q$ is a countable dense subgroup of $G$, in the box topology, is also hyperconnected, in particular, connected. This is a step to answer a question 
posed by Chatyrko and Karassev [CK]: Let $X_{\alpha}, \alpha \in A$, be an infinite system of nondegenerated connected Hausdorff spaces. Under what conditions on the system is the space $\prod_{\alpha \in A}^{b} X_{\alpha}$ (dis)connected?

We also study the relationship (inclusion or equality) between the topologies $\tau\left(Q_{1}\right)$ and $\tau\left(Q_{2}\right)$, when $Q_{1}$ and $Q_{2}$ are countable dense subgroups of $G$ such that $Q_{1} \subseteq Q_{2}$. We proved that if the factor group $Q_{2} / Q_{1}$ is finite then $\tau\left(Q_{1}\right) \subseteq \tau\left(Q_{2}\right)$, and that if the factor group $Q_{2} / Q_{1}$ is infinite then $\tau\left(Q_{1}\right) \nsubseteq \tau\left(Q_{2}\right)$. Moreover, we prove that for any given countable dense subgroups $Q_{1}$ and $Q_{2}$ of $G$ the topological spaces $G\left(Q_{1}\right)$ and $G\left(Q_{2}\right)$ are homeomorphic.

\section{Paper V: Sets with the Baire property in topologies formed from a given topology and ideals of sets}

Let $X$ be a non-empty set and let $\tau_{1}, \tau_{2}$ be topologies on $X$. It is well known that the family of sets with the Baire property in $\left(X, \tau_{i}\right)$ contains all open sets and all meager sets of $\left(X, \tau_{i}\right), i=1,2$. However, depending on the topology $\tau_{i}$, the family of sets with the Baire property in $\left(X, \tau_{i}\right)$ can contain or not contain every subset of $X$. This implies that neither the family of sets with the Baire property in $\left(X, \tau_{1}\right)$ nor the family of sets with the Baire property in $\left(X, \tau_{2}\right)$ needs to contain the other.

One can pose the following question: What relationship between the topologies $\tau_{i}, i=1,2$ implies a relationship (inclusion or equality) between the families of sets with the Baire property in $\left(X, \tau_{i}\right), i=1,2$ ?

In this paper, we focus on answering this question, with a particular attention to the case in which the topology $\tau_{2}$ is formed with the help of a local function defined by the topology $\tau_{1}$ and an ideal of sets on $X$.

We first introduce a concept generalizing the notion of admissible extension, that we call the $\pi$-compatibility. Two topologies $\tau_{1}$ and $\tau_{2}$ on $X$ are said to be $\pi$-compatible if for each non-empty element $O \in \tau_{2}$ there is a non-empty element $V \in \tau_{1}$ which is a subset of $O$, and vice versa. We prove that if $\tau_{1}$ and $\tau_{2}$ are $\pi$-compatible then the spaces $\left(X, \tau_{1}\right)$ and $\left(X, \tau_{2}\right)$ have the same families of sets with the Baire property.

We next consider the case where $\tau_{2}$ is defined from $\tau_{1}$ and an ideal of sets $\mathscr{I}$ on $X$, that is, $\tau_{2}=\tau_{1}^{*}(\mathscr{I})$. For this case, we study conditions under which $\tau_{1}^{*}(\mathscr{I})$ is an admissible extension of $\tau_{1}$. We prove that if $\mathscr{I}$ is a subideal of nowhere dense sets in $\left(X, \tau_{1}\right)$ then $\tau_{1}^{*}(\mathscr{I})$ is an admissible extension of $\tau_{1}$ on $X$, and hence the spaces $\left(X, \tau_{1}\right)$ and $\left(X, \tau_{1}^{*}(\mathscr{I})\right)$ have the same families of sets with the Baire property. In addition, we prove that if $\left(X, \tau_{1}\right)$ is a second countable topological space and $\mathscr{I}$ is a $\sigma$-subideal of meager sets in $\left(X, \tau_{1}\right)$ such that $\tau_{1} \cap \mathscr{I}=\{\emptyset\}$ then the spaces $\left(X, \tau_{1}\right)$ and $\left(X, \tau_{1}^{*}(\mathscr{I})\right)$ have the same families of sets with the Baire property. 
Several other conditions implying a relationship between sets with the Baire property in $\left(X, \tau_{1}\right)$ and sets with the Baire property in $\left(X, \tau_{1}^{*}(\mathscr{I})\right)$ are derived. We also provide conditions under which different ideals of sets produce the same topologies on $X$.

We note that a similar question can be posed for any other families of sets obtained by using the topologies, and the opposite question can be also posed. In this paper, we also consider the question for nowhere dense sets and meager sets. However, we observe that the results for sets with the Baire property do not always carry over nicely to nowhere dense sets and to meager sets. 



\section{Bibliography}

[ACN] M. Aigner, V. A. Chatyrko and V. Nyagahakwa, On countable families of sets without the Baire property, Colloquium Mathematicum, 2013, Vol.133, No.2, 179 - 187.

[Ch1] V. A. Chatyrko, On Vitali sets and their unions, Matematicki Vesnik, 2011, Vol.63, No.2, 87 - 92 .

[Ch2] V. A. Chatyrko, On countable unions of nonmeager sets in hereditarily Lindelöf spaces, p-Adic Numbers, Ultrametric Analysis, and Applications, 2011, Vol.3, No.1, 1 - 6 .

$[\mathrm{CH}]$ V. A. Chatyrko and Y. Hattori, A poset of topologies on the set of real numbers, Comment. Math. Univ. Carolin., 2013, Vol.54, No.2, 189 - 196.

[CN] V. A. Chatyrko and V. Nyagahakwa, On the families of sets without the Baire property generated by the Vitali sets, p-Adic Numbers, Ultrametric Analysis, and Applications, 2011, Vol.3, No.2, 100 - 107.

[CK] V. A. Chatyrko and A. Karassev, The (dis)connectedness of products in the box topology, Questions and Answers in General Topology, 2013, Vol.31, No.1, $1-21$.

[En] R. Engelking, Theory of dimensions, finite and infinite, Sigma series in pure mathematics, Volume 10, Heldermann Verlag, 1995.

[FK] M. Foreman and A. Kanamori, Handbook of set theory, Volume 1, Springer Science + Business Media B.V., 2010. 
[GW] D. M. Gabbay, J.H. Woods and A. Kanamori, Handbook of the history of logic: Sets and extensions in the twentieth century, Vol.6, Elsevier, 2012.

[Ha] E. Hayashi, Topologies defined by local properties, Math. Ann., 1964, Vol.156, No.3, 205 - 215.

[HR] E. Hewitt, K.A Ross, Abstract harmonic analysis: Structure of topological groups, Integration theory, Group representations, Volume I, Springer-Verlag Berlin Heidelberg, 1963.

[Ht] Y. Hattori, Order and topological structures of poset of the formal balls on metric spaces, Mem.Fac.Sci.Eng. Shimane Univ.Ser. B: Math. Sci., 2010, Vol. 43, 13-26.

[JH] D. Janković and T.R. Hamlett, New topologies from old via Ideals, Amer. Math. Monthly, 1990, Vol.97, No.4, 295 - 310.

[KF] A. N Kolmogorov and S.V Fomin, Introductory real analysis, Courier Dove Publications, 1975.

[Kh1] A.B. Kharazishvili, Nonmeasurable sets and functions, Vol.195, Elsevier, 2004.

[Kh2] A.B Kharazishvili, Measurability properties of Vitali sets, The American Mathematical Monthly, 2011, Vol.118, No.8, 693 - 703.

[Ku] K. Kuratowski, Topology, Volume I, PWN, Warszawa and Academic Press, New York, 1966.

[Mo] S. A. Morris, Pontryagin duality and the structure of locally compact abelian groups, Cambridge University Press, 1977.

[N] V. Nyagahakwa, Semigroups of sets without the Baire property in finite dimensional Euclidean spaces, Licentiate thesis, Linköping University, 2015.

[Ox] J.C. Oxtoby, Measure and Category: Graduate texts in mathematics, Springer-Verlag, New York, Heldenberg, Berlin, 1971.

[So] R. M. Solovay, A model of set theory in which every set of reals is Lebesgue measurable, Ann. Math., 1970, Vol. 92, No.1, 1 - 56.

[Vi] G. Vitali, Sul problema della misura dei gruppi di punti di una retta, Bologna, Italy, 1905. 


\section{Papers}

The articles associated with this thesis have been removed for copyright reasons. For more details about these see:

http://urn.kb.se/resolve? urn:nbn:se:liu:diva-137057 